\title{
Addressing Phase Transitions in Wireless Networking Optimization
}

\author{
Maria Michalopoulou and Petri Mähönen* \\ RWTH Aachen University, Institute for Networked Systems \\ $\{$ mmi, pma\}@inets.rwth-aachen.de
}

\begin{abstract}
The general aim of this paper is to introduce the notion of phase transitions into wireless networking optimization. Although the theory of phase transitions from statistical physics has been employed in optimization theory, phase transitions in the context of optimization of wireless networks have not yet been considered. In wireless networking optimization, given one or more optimization objectives we often need to define mathematically an optimization task, so that a set of requirements is not violated. However, especially recent trends in wireless communications, such as self-organized networks, femto-cellular systems, and cognitive radios, calls for optimization approaches that can be implemented in a distributed and decentralized fashion. Thus we are interested to find utility-based approaches that can be practically employed in a self-organizing network. We argue that phase transitions can be identified and taken appropriately into account in order to eliminate the emergence of undesirable solutions that lie near the point where the phase transition occurs. As an example we present a simple power control problem for a macrocell-femtocell network scenario. We formulate a distributed framework of the problem where we model a phase transition effect by means of a dummy variable in order to exclude solutions lying in the one side of the phase transition.
\end{abstract}

\section{Introduction}

The concept of optimization - not only in its strict mathematical sense - is fundamental in wireless networking research. Especially the trend towards cognitive and self-organizing wireless networks has raised the demand to dive deeper into distributed network optimization.

Strictly speaking, optimization is simply the procedure of maximizing some benefit while minimizing a well-defined cost. However, we would like to stress that in wireless networking the term optimization is used rather more freely. Generally, in engineering sciences the aim of optimization is often relaxed to a more realistic design goal, that is to obtain a required performance level while

\footnotetext{
* The authors would like to thank the RWTH Aachen University and the German Research Foundation (Deutsche Forschungsgemeinschaft, DFG) for financial support through the UMIC research center. We also thank the European Union for providing partial funding of this work through the FARAMIR project.
} 
satisfying some constraints. Especially in self-organizing wireless networks where decentralized and distributed approaches are called for, the standard definition of an optimization problem by means of a single optimization function that expresses the overall system performance is not practically useful. Instead, a distributed framework is needed, where each network node aims to optimize its own individual utility function.

The general aim of this paper is to introduce the notion of phase transitions into wireless networking optimization. Our initial motivation emerges from the fact that phase transitions are already exploited in optimization theory and have proven to be a very powerful tool in modeling dynamical and statistical physics systems (see, for example, [1-4] ). It has been recognized that threshold phenomena, analogous to phase transitions in physical systems, occur in several optimization problems. Actually, there is a considerable and increasing amount of literature that attempts to draw a connecting line between optimization problems and the theory of phase transitions from statistical physics. Despite that, the notion of phase transition has not yet been considered in wireless networking optimization. There is a limited amount of literature addressing phase transitions occurring in wireless networks from a rather theoretical perspective (see, for example, [5 9]), but to the best of our knowledge phase transitions have not been addressed in the context of wireless networking optimization problems.

We argue that the study of phase transitions in wireless networking optimization problems is important not only from a theoretical point of view, but can be also useful in attacking practical optimization problems in a more comprehensive way. A phase transition corresponds to a large, abrupt deviation in the behavior of the problem, and in most cases this change has a significant effect in the performance as well. Taking into account the highly dynamic characteristics of wireless networks we would like then to end up with a solution that lies sufficiently away from a point where a phase transition occurs, so that a small drift from the obtained solution will not cause the system to undergo a phase transition. Therefore, a phase transition can be identified and taken into account in constructing a solution methodology, or even in defining the mathematical representation of the problem in a way that the emergence of undesirable solutions, that lie near a phase transition point, is eliminated.

As a case study we demonstrate a power control optimization problem in a macrocell-femtocell network scenario. More specifically, we suggest that in many cases a hard optimization constraint can be interpreted as a phase transition effect since it defines a threshold that usually corresponds to a qualitative change in the system. We show how such phase transition effects can be mathematically incorporated by means of a dummy variable; this is a typical technique used in econometrics for modeling qualitative changes. In our scenario we apply this approach in formulating a distributed, utility-based representation of the problem in order to prevent the occurrence of poor solutions.

The rest of the paper is organized as follows. In Section 2 we elaborate on the main idea and motivation behind this work. In Section 3 we introduce our example scenario; in Subsection 3.1 we present the system model and the phase 
transition arising from the standard-form formulation of the optimization problem, and in Subsection 3.2 we discuss the distributed, utility-based approach of the problem. Finally, the paper is concluded in Section 4 .

\section{Motivation for Taking Phase Transitions into Account}

The notion of phase transitions originates in physics and has been extensively studied, particularly in the field of statistical mechanics [10]. A system can exist in a number of different phases, which can be distinguished from each other by a set of suitably chosen and well-defined parameters. The macroscopic properties of a system in different phases usually differ significantly. A phase transition is a transition of the system from one phase to another caused by a small change of an external parameter, called control parameter. More specifically, there is a well-defined value of the control parameter, termed critical point, above which one phase exists and as the control parameter goes below that value a new phase appears. Briefly, a phase transition is an abrupt and qualitative change in the macroscopic properties of the system. One of the most widely known examples is the liquid-gas phase transition, where temperature plays the role of the control parameter under the assumption of constant pressure. A phase transition is also characterized by an order parameter. The order parameter is usually a quantity which is zero in one phase (usually above the critical point), and obtains a nonzero value as the system enters the new phase.

There is an inherent connection between statistical physics and optimization theory. After all, the main task in an optimization problem is to find a configuration of the problem parameters that minimizes a cost function, which is analogous to finding the minimal energy state in statistical physics. Within the context of optimization problems a phase transition is simply an abrupt change in the behavior of the problem. The identification of an existing phase transition contributes to a better understanding of the problem that is not only of theoretical interest, but can be also useful in solving optimization problems more competently.

However, optimization tasks in wireless networking scenarios constitute a special case due to the dynamic characteristics of the wireless environment. Unlike classical optimization problems, a wireless networking optimization task is executed on a wireless network which is not isolated from the outside world, meaning that the system we have to deal with is subject to external influences. Consequently, finding an optimal or a sufficiently good solution is not always enough as in the case of classical optimization of static systems because in a wireless network several factors might easily cause the system to drift from the solution after it has been reached. Under these dynamic conditions not only the optimality of the solution, but also the stability of the performance is an important factor that has to be taken into account. The behavior of the system around a selected solution point needs to be considered as well. As discussed above a phase transition is synonymous to an abrupt change in the behavior; if a phase transition is located near to a selected solution point, then a slight drift from this point 
might lead to a large undesirable fluctuation in the system performance. Under these circumstances, even the optimal solution might be rather undesirable if it is highly unstable in terms of performance. Therefore, if a phase transition is likely to cause a large fluctuation affecting the performance of the network, this fact has to be taken into account in solving the optimization problem. For instance, the solution can be restricted to lie somewhat away from the phase transition point. A general rule regarding whether a phase transition should be taken into account when solving a problem and how it should be treated cannot be established, but a different decision has to be made each time depending on the behavior of the specific problem under study.

Let us consider, for example, an optimization problem with one or more constraints, among which an inequality constraint. The inequality constraint can be addressed as a phase transition that separates the solution space of the problem into two phases, one that corresponds to the subset of feasible solutions and a second phase that corresponds to the unacceptable solutions. Therefore, solving the problem very close to the critical point, which is defined by the value of the inequality constraint, might result in experiencing fluctuations that can easily bring the system into the unacceptable performance regime. However, we need to stress that the appropriate treatment for this phase transition can only be determined according to problem-specific factors. For example, in several cases falling slightly below the value defined by an inequality constraint corresponds to an analogous performance degradation which brings the system at an operational point not satisfying the system requirements. In such a case we might decide that slight violations of the inequality constraint for short time periods can be tolerated. Naturally, a critical factor that we need to take into account in this decision is how sharp is the fluctuation effect induced by the phase transition, that is how drastic are the behavioral changes we experience due to small deviations of the control parameter around the phase transition point. On the other hand, sometimes the inequality constraint might correspond to a point below which the system collapses. In these cases we should select an approach that will guarantee that the constraint will not be violated, i.e, the solution will lie sufficiently away from the phase transition point.

The phase transition dynamics and analysis we are introducing can be seen as a complementary, or sometimes alternative tool, for the better known game theoretical analysis of Nash equilibrium. Phase transitions in the context of utility optimization provides different mathematical framework from game theory, and in a sense it emphasizes the need to analyze the stability of optimal solutions and consequences of perturbations around those points.

\section{Introducing the Notion of Phase Transitions in a Power Control Scenario}

We demonstrate the main idea of the paper by introducing the notion of phase transitions in a power control optimization problem. More specifically we consider an uplink power control problem in a two-tier femtocell network. This 
scenario has become really very interesting due to the fact that femtocells offer an attractive possibility to increase the capacity of wireless networks. We stress that our aim in this paper is not to present a novel solution methodology for the specific power optimization task, but rather to propose a different and complementary optimization approach - based on the notion of phase transitions - that can be used to address a multitude of other wireless networking optimization problems as well.

\subsection{The System Model}

We consider a macrocell-femtocell scenario where a single Macrocell Base Station (MBS) is underlaid with $M$ Femtocell Access Points (FAPs). The system model is illustrated in Figure 1. The macrocell and the femtocells have access

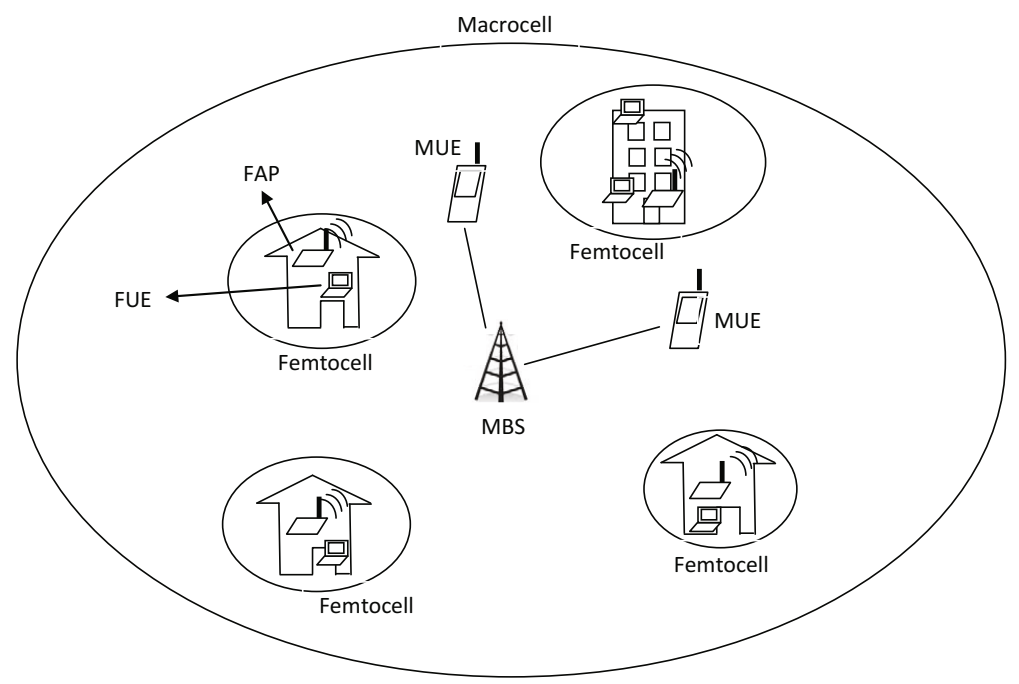

Fig. 1. A two-tier femtocell network where a cellular macrocell is underlaid with shorter range femtocells

to the same set of $K$ subchannels, $\{0,1, \ldots, k, \ldots, K-1\}$. The cellular network operator owning the MBS is the so-called licensed user of the spectrum, whereas the femtocells - which are small, low-power networks deployed in homes and enterprises - are allowed to make use of the same channels (frequencies) under the strict requirement that they will not disrupt the operation of the macrocell, i.e., a specified Signal to Interference-plus-Noise Ratio (SINR) for the macrocell communications needs to be guaranteed anytime. We assume that a subchannel allocation scheme operates independently of power control. After the subchannel assignment a power control scheme can be applied independently in each subchannel. Therefore, without loosing generality, we shall be concerned with the 
uplink power control in a single subchannel, $k$. We suppose that a subset of $N$ femtocells, $\{0,1, \ldots, n, \ldots, N-1\}$, are assigned with subchannel $k$. Each femtocell might serve several users, but obviously only one user will use subchannel $k$ in each of the $N$ femtocells. Therefore, we will concentrate only on a single Femtocell User Equipment (FUE) in each femtocell $n$, denoted as $F U E_{n_{k}}$. We suppose that the subchannel $k$ is currently used also by the MBS in order to serve a Macrocell User Equipment, $M U E_{k}$. This fact introduces a macrocell-femtocell interference in our scenario, whereas the reuse of subchannel $k$ by more than one femtocells causes a femtocell-to-femtocell interference. Since we concentrate only on subchannel $k$, for the sake of brevity the subscript $k$ shall be omitted in the rest of the paper.

Assuming that the transmission power in the macrocell is already determined, the goal is to find the uplink transmission powers, $\left\{p_{1}, p_{2}, \ldots, p_{n}, \ldots, p_{N}\right\}$, of the FUEs in each of the $N$ femtocells so that the total femtocell capacity, that is the sum of the capacities achieved in each of the $N$ femtocells, is maximized. Thus, the objective function can be defined as follows:

$$
\max _{p_{n}} \sum_{i=1}^{N} C_{n},
$$

where $C_{n}$ is the capacity achieved in femtocell $n$.

Clearly two types of interference constraints arise in the uplink case; one accounts for the interference suffered by the MBS from the FUEs, and the second for the interference suffered by a FAP from the MUE and from FUEs in neighboring femtocells. However, the first priority of a cellular operator is to fully satisfy all macrocell users, and shall allow the deployment of cochannel femtocells only if they do not disrupt the communication within the macrocell. Thus, we can address the interference constraints by borrowing the concept of primary and secondary users from Cognitive Networks [1]; the MBS plays the role of a primary user, whereas the FAPs are the secondary users. We apply this concept by defining a hard constraint for the SINR at the MBS, and soft constraints for the SINRs of the FAPs as follows

$$
\begin{aligned}
& \gamma_{M} \geq \gamma_{M_{\text {target }}} \quad \text { (Hard Constraint) } \\
& \gamma_{F_{n}} \geq \gamma_{F_{m i n}}, \forall n \in\{1,2, \ldots, n, \ldots N\} \quad \text { (Soft Constraint) }
\end{aligned}
$$

In optimization theory a hard constraint is a constraint that must be satisfied. On the other hand, soft constraints are supposed to be followed to the extent that this is possible, but not at the expense of the hard constraints.

Although femtousers will not be necessarily fully satisfied, a soft SINR constraint for every FAP aims to preserve the bit rate in all the femtocells at an acceptable level in order to avoid an extremely unfair capacity assignment among the femtocells. On the other hand the macrouser must remain fully satisfied all the time, meaning that a violation of the hard constraint (Equation 2) is not tolerated. The hard constraint can be translated into a phase transition separating two phases in the solution space of the optimization problem, one corresponding 
to feasible solutions, and the other to unacceptable solutions. The received SINR at the MBS acts as the control parameter of the phase transition and the target value defined by the corresponding constraint, $\gamma_{M_{\text {target }}}$, is the critical value.

Therefore, we need to take care that a solution algorithm for this particular scenario shall not converge to a solution which is too close to the phase transition point. In this case the critical point is precisely known, thus if we plan to solve the problem in a centralized fashion (based on Equations 10 2 and 3) we can just shift the critical point in order to include a safety margin, $\epsilon$, by slightly modifying the corresponding constraint (Equation 2) as follows

$$
\gamma_{M} \geq \gamma_{M_{\text {target }}}+\epsilon
$$

Naturally, the value of the margin, $\epsilon$, has to be determined according to the severeness of the performance fluctuations around the critical point.

Nevertheless, in practice the solution to this issue is usually less trivial. In the following subsection we will be discussing a self-organizing, distributed approach for this scenario.

\subsection{A Utility-Based Distributed Approach}

In the previous subsection we discussed the occurrence of a phase transition in an uplink power control problem for a macrocell-femtocell scenario. The definition of the optimization problem as presented in Section 3.1 corresponds to a centralized approach of the problem and thus is not particularly attractive from a more practical point of view. In this section we shall consider a distributed, self-organizing approach of the problem based on utility functions. The communicating pairs of FAPs and FUEs are responsible to determine their own transmission powers. For this purpose we assume that the elements of each pair exchange control information between them. Specifically, for the case of uplink power control the task is to determine the transmission powers of each FUE and we suppose that the corresponding FAP is informing the FUE about its received SINR.

The utility function of each FUE consists of a reward term, which assigns a payoff increasing with the SINR achieved at the FBS, and a penalty term, which penalizes the interference caused by the FUE to other FAPs of neighboring femtocells, and to the MBS. The reward term corresponds to the objective function of the optimization problem (Equation 1), and the penalty term accounts for the constraints (Equations 2 and 3). Additionally, a variable $\beta$ needs to be introduced in order to control the interaction between the reward term and the penalty. Therefore, we write the utility function for $F U E_{n}$ as follows

$$
U_{n}=R_{n}\left(\gamma_{F_{n}}, \gamma_{F_{m i n}}\right)-\beta P_{n}\left(p_{n}, \gamma_{F_{n}}\right) .
$$

We define the reward function as follows [12]

$$
R_{n}\left(\gamma_{F_{n}}, \gamma_{F_{m i n}}\right)=1-e^{\left(-\alpha\left(\gamma_{F_{n}}-\gamma_{F_{m i n}}\right)\right)}
$$


and the penalty term as

$$
P_{n}\left(p_{n}, \gamma_{F_{n}}\right)=p_{n} \gamma_{F_{n}}
$$

The reward is negative if the achieved SINR is below the target SINR, it becomes 0 when the SINR is equal to the target SINR and increases exponentially towards 1. The rate of this exponential increase is controlled by parameter $a$. On the other hand, the penalty term discourages the FUE to increase its transmission power unlimitedly by assigning a high cost if the transmission power and the achieved SINR are high. We shall be considering one of the simplest strategies that can be adopted. The FUEs initialize their transmission powers at some minimum value, $p_{\text {min }}$, and continue by increasing the transmission power in small steps if such a decision will increase their utility function.

The quantitative results that shall be presented in the following discussion are obtained by a Matlab simulation of this distributed, utility-based scheme. We consider a MBS and an outdoor MUE separated by a distance equal to 250 meters. Then, we consider that $50 \mathrm{FBSs}$ use channel $k$ within the coverage area of the MBS and are placed at random locations within 300 meters from the MBS. The FUEs are within a radius of 20 meters from their FBS, and the FBSs are at least 40 meters away from each other (i.e., we assume that the coverage areas of FBSs do not overlap with each other). All FUEs are considered to be indoors, in the same building with their associated FBS, but within a different building from neighboring FBSs. The pathloss models we use follow the 3GPP LTE (Long Term Evolution) specification [13]. We also consider lognormal shadowing with standard deviation equal to $4 \mathrm{~dB}$ for communication within a femtocell, and $8 \mathrm{~dB}$ for every other case [13]. We consider Additive White Gaussian Noise (AWGN) with variance $\sigma^{2}=N_{o} B$, where $N_{o}$ is the noise power spectral density and $B$ is the subchannel bandwidth. The noise power spectral density is set to -174 $\mathrm{dBm} / \mathrm{Hz}$ and the subchannel bandwidth is $200 \mathrm{KHz}$.

The solution where the network will converge is determined by the value of the parameter $\beta$. As already discussed, the femtocells should not be deployed at the expense of the macrocell performance, hence a violation of the hard constraint of Equation 2 is not tolerated. Consequently, an appropriate selection of $\beta$ must guarantee the satisfaction of this constraint. Figure 2 illustrates the achieved SINR at the MBS for different values of $\beta$. The different curves correspond to results obtained for different network topologies. Clearly, the outcome is highly variable for different random topologies indicating that the determination of the parameter $\beta$ is definitely not a straightforward task. However, in both cases the SINR increases approximately linearly with $\beta$. Actually, in practice the SINR requirements are imposed by the data rate required to provide the services accessed by the user. From the user's point of view the achieved data rate is the performance. If we plot the achieved capacity based on the Shannon's law instead of the SINR (Figure 3) we observe that the relation with $\beta$ is not linear in this case and for the lower values of $\beta$ the performance in terms of capacity is even more sensitive to small changes of $\beta$. Therefore, there is a region where even small errors in the determination of an appropriate value for $\beta$ can affect 


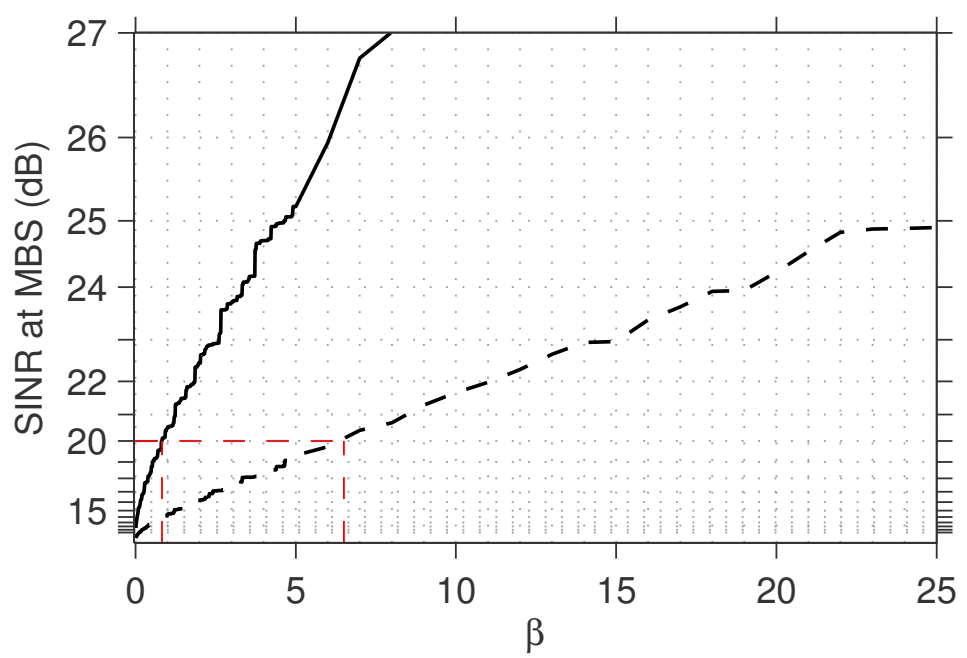

Fig. 2. The SINR received at the MBS (linear scale) against the parameter $\beta$ for two different random topologies. The target SINR at the MBS $\left(\gamma_{M_{\text {target }}}\right)$, i.e., the critical point, is set to $20 \mathrm{~dB}$.

drastically the macrocell capacity. The dashed red lines in Figure 3 indicate the values corresponding to the critical SINR value of $20 \mathrm{~dB}$ at the MBS.

In utility-based approaches it is a common tactic to account for inequality constraints by introducing penalty terms. Nevertheless, the two representations are not precisely the same; the penalty term cannot define a precise threshold like an inequality constraint. As discussed in Section 3.1. the hard inequality constraint of Equation 2 defines basically the critical point between two phases, one corresponding to the acceptable and the other to unacceptable solutions. Although this phase transition in the solution space is quite straightforward from the standard constraint-based representation of the optimization problem, this effect is not clearly mapped in the distributed approach. More precisely, what is missing from the utility-based approach is the effect of a drastic degradation of the utilities as the SINR at the MBS crosses the critical point, that will model the phase transition from acceptable to unacceptable solutions. Towards this direction, we shall create this phase transition effect by means of a binary variable. The usage of binary (or dummy) variables to model structural changes 1 is commonly employed in econometrics [14]. We define the utility functions as follows

$$
U_{n}=R_{n}\left(\gamma_{F_{n}}, \gamma_{F_{m i n}}\right)-(\beta+\delta) P_{n}\left(p_{n}, \gamma_{F_{n}}\right)
$$

\footnotetext{
${ }^{1}$ In econometrics terminology such phenomena are called structural changes, but they are clearly analogous to what we call phase transitions.
} 


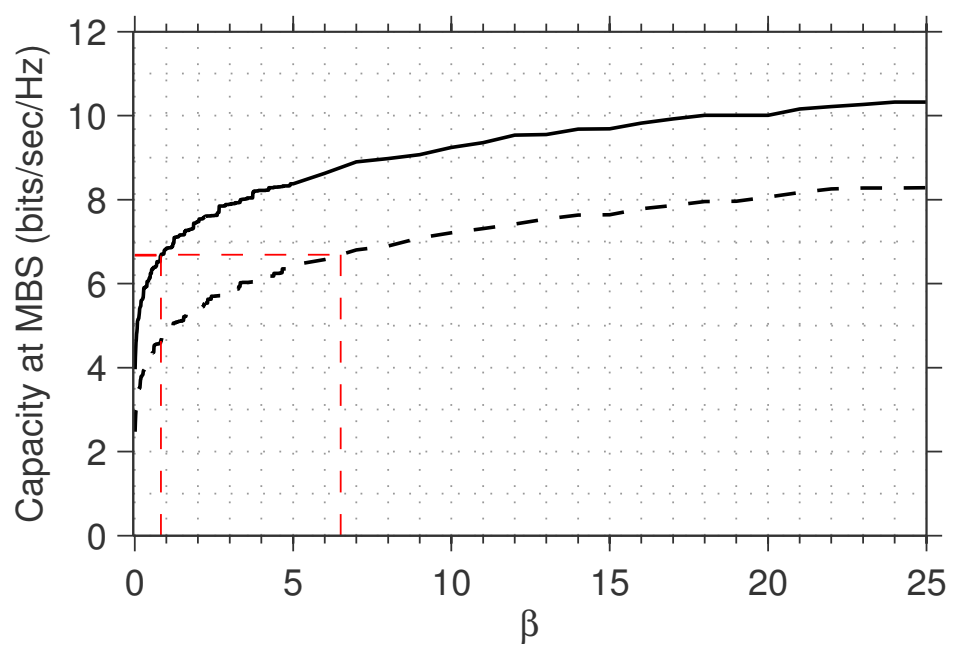

Fig. 3. The normalized capacity for the MBS against the parameter $\beta$ for four different network topologies. The dashed red lines indicate the values corresponding to the critical SINR value of $20 \mathrm{~dB}$ at the MBS.

The binary variable is the parameter $\delta$, which can be defined as follows

$$
\delta=\left\{\begin{array}{l}
x, \text { if } \gamma_{M}<\gamma_{M_{\text {target }}}-\epsilon \\
0, \text { otherwise }
\end{array}\right.
$$

where $x$ is a very large value which makes the penalty very expensive if the SINR at the MBS is below the critical value, so that a further increase of the transmission powers is prohibited. Initially, the FUEs set the parameter $\delta$ to 0 . The MBS shall inform all the FUEs if its received SINR is below the threshold value, and then the FUEs shall change the value of $\delta$ to $x$. The parameter $\epsilon$ is just a safety margin from the critical point. Figure 4 illustrates the SINR at the MBS when using the utility function of Equation 8 for exactly the same configurations as those used to produce Figures 2 and 3 . Basically, the binary variable $\delta$ does not allow the system to undergo a phase transition towards the regime of unacceptable solutions.

Finally, we would like to stress that the method of introducing a binary variable in order to model an existing phase transition is not specific to this particular scenario presented here. In general, a binary variable can be used to model a discrete shift, or equivalently, it can distinguish two alternatives. Therefore, the proposed approach of introducing a binary variable in modeling a utility function is in general suitable for treating two phases differently like, for example, in case we want to favor a solution lying in the one side of a phase transition. 


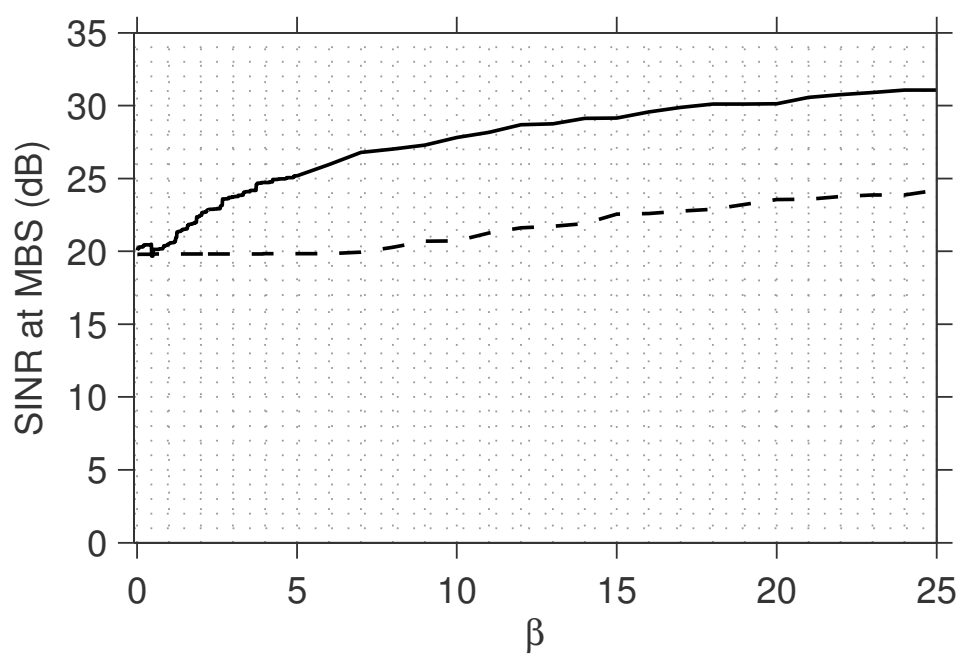

Fig. 4. The SINR received at the MBS against the parameter $\beta$ after introducing the binary variable $\delta$ in the utility functions

\section{Conclusions}

In this paper we addressed phase transitions in the context of wireless networking optimization. A phase transition is often translated into an abrupt and drastic change in the behavior of an optimization problem. Especially in wireless networking, such effects can result in a significant performance instability because a network might easily drift from a solution point due to the dynamic environment and conditions. Therefore, we argue that in several cases the awareness of a phase transition should be taken into account when solving the problem in order to ensure the stability of the solution.

As a case study we presented a power control optimization problem for a macrocell-femtocell network. The specific scenario exhibits a phase transition between two regions of the solution space, one corresponding to acceptable and the other to unacceptable solutions. We showed how we can formulate a distributed, utility-based approach of the optimization problem in order to map clearly this effect of distinguishing between a favorable and undesirable phase by means of a binary variable. This approach is suitable if we want to treat two phases in a different way, for instance, if we want to favor solutions lying in the one side of a phase transition.

\section{References}

1. Hartmann, A., Weigt, M.: Phase Transitions in Combinatorial Optimization Problems. Wiley (2005) 
2. Monasson, R., Zecchina, R., Kirkpatrick, S., Selman, B., Troyansky, L.: Determining computational complexity from characteristic 'phase transitions'. Nature 400(6740), 133-137 (1999)

3. Martin, O., Monasson, R., Zecchina, R.: Statistical mechanics methods and phase transitions in optimization problems. Theor. Comput. Sci. 265, 3-67 (2001)

4. Achlioptas, D., Naor, A., Peres, Y.: Rigorous location of phase transitions in hard optimization problems. Nature 435, 759-764 (2005)

5. Krishnamachari, B., Wicker, S., Bejar, R.: Phase transition phenomena in wireless ad hoc networks. In: Global Telecommunications Conference, GLOBECOM 2001, vol. 5, pp. 2921-2925. IEEE (2001)

6. Krishnamachari, B., Wicker, S., Bejar, R., Pearlman, M.: Critical Density Thresholds in Distributed Wireless Networks, pp. 279-296. Kluwer Academic Publishers, USA (2003)

7. Durvy, M., Dousse, O., Thiran, P.: Border effects, fairness, and phase transition in large wireless networks. In: The 27th Conference on Computer Communications, INFOCOM 2008, pp. 601-609. IEEE (April 2008)

8. Xing, F., Wang, W.: On the critical phase transition time of wireless multi-hop networks with random failures. In: Proc. of the 14th ACM International Conference on Mobile Computing and Networking, MobiCom 2008, pp. 175-186 (2008)

9. Michalopoulou, M., Mähönen, P.: Game theory for wireless networking: Is a nash equilibrium always a desirable solution? In: 2012 IEEE 23rd International Symposium on Personal Indoor and Mobile Radio Communications (PIMRC), pp. 1249-1255 (September 2012)

10. Reichl, L.: A Modern Course in Statistical Physics. Wiley (1998)

11. Broderson, R., Wolisz, A., Cabric, D., Mishra, S., Willkomm, D.: Corvus: A cognitive radio approach for usage of virtual unlicensed spectrum. White Paper (2004)

12. Chandrasekhar, V., Andrews, J., Muharemovic, T., Shen, Z., Gatherer, A.: Power control in two-tier femtocell networks. IEEE Transactions on Wireless Communications 8(8), 4316-4328 (2009)

13. 3GPP TR36.814: Further advancements for E-UTRA, physical layer aspects. Technical report (March 2010), http://www.3gpp.org/

14. Greene, W.: Econometric Analysis. Prentice Hall (2003) 